\title{
Evaluation of Adhesion of Hydroxyapatite Films Fabricated on Biomedical $\beta$-Type Titanium Alloy after Immersion in Ringer's Solution
}

\author{
Junko Hieda ${ }^{1, * 1}$, Mitsuo Niinomi ${ }^{2}$, Masaaki Nakai ${ }^{2}$, Ken $\mathrm{Cho}^{2}$ and Ayaka Matsubara ${ }^{3, * 2}$ \\ ${ }^{1}$ Graduate School of Science and Engineering, Tokyo Institute of Technology, Tokyo 152-8552, Japan \\ ${ }^{2}$ Institute for Materials Research, Tohoku University, Sendai 980-8577, Japan \\ ${ }^{3}$ Graduate School of Engineering, Tohoku University, Sendai 980-8579, Japan
}

\begin{abstract}
The adhesive strengths between sol-gel fabricated hydroxyapatite (HAp) films and Ti-29Nb-13Ta-4.6Zr alloy (TNTZ) were evaluated before and after immersion in Ringer's solution at $310 \mathrm{~K}$ for $7 \mathrm{~d}$. The effect of the surface morphology of TNTZ on the adhesion of HAp films was also investigated. The fracture surfaces after adhesion tests were observed and fracture areas at the interface between HAp films and TNTZ were also evaluated before and after immersion in Ringer's solution. No significant differences in the adhesive strengths between HAp films and TNTZ disks with mirror-polished and mechanically polished surfaces were found. The fracture area at the interface between HAp film and mirror-polished TNTZ disk increases after immersion in Ringer's solution, whereas no fracture occurs at the interface between HAp film and mechanically polished TNTZ disk before and after immersion in Ringer's solution. It is supposed that Ringer's solution penetrates along the cracks that are formed in the HAp films during calcination and the regions with weak adhesion between HAp and TNTZ are connected during immersion in Ringer's solution in the case of HAp films fabricated on mirror-polished TNTZ. From these results, it is found that a relatively rougher surface is effective in improving the adhesion of HAp films fabricated on TNTZ before and after immersion in Ringer's solution. [doi:10.2320/matertrans.M2015223]
\end{abstract}

(Received June 2, 2015; Accepted July 21, 2015; Published September 25, 2015)

Keywords: $\beta$-type titanium alloy, hydroxyapatite, surface morphology, adhesive strength, Ringer's solution

\section{Introduction}

Titanium (Ti) and Ti alloys have been commonly used as materials for biomedical applications, such as bone prosthesis and dental implants, because of their good mechanical properties, high corrosion resistance, and biocompatibility in comparison to other metallic biomaterials. ${ }^{1,2)}$ Especially, Young's modulus is a very important factor when $\mathrm{Ti}$ and $\mathrm{Ti}$ alloys are employed for load bearing implants. The higher Young's modulus of metallic biomaterials than that of the bone gives rise to the stress shielding effect that is the phenomenon of bone weakening because the load transfers preferentially through the implant made of the metallic biomaterials. $^{1,2)}$ The $\beta$-type Ti alloy $\mathrm{Ti}-29 \mathrm{Nb}-13 \mathrm{Ta}-4.6 \mathrm{Zr}$ alloy (TNTZ) has been developed in order to achieve a low Young's modulus $(\sim 60 \mathrm{GPa}){ }^{3)}$ The Young's modulus of TNTZ is lower than that of stainless steel $(\sim 180 \mathrm{GPa})$ and Ti6Al-4V ELI $(\sim 110 \mathrm{GPa})$, which are conventional alloys for biomedical applications. ${ }^{4)}$ In addition, TNTZ consists of nontoxic and non-allergenic elements. Thus, TNTZ is a very suitable Ti alloy for implant devices.

Although $\mathrm{Ti}$ and $\mathrm{Ti}$ alloys exhibit excellent hard-tissue compatibility attributed to osseointegration, ${ }^{5,6)}$ it takes a long time to achieve the remodeling of the bone on the surface of $\mathrm{Ti}$ and $\mathrm{Ti}$ alloys. Therefore, to improve the hard-tissue compatibility of $\mathrm{Ti}$ and $\mathrm{Ti}$ alloys, various surface treatments have been developed for the formation of inorganic layers which exhibit the desired high hard-tissue compatibility. The most common way for providing the high hard-tissue compatibility is coating of hydroxyapatite (HAp: $\mathrm{Ca}_{10}\left(\mathrm{PO}_{4}\right)_{6-}$ $\left.(\mathrm{OH})_{2}\right)$ on the surface of metal, which is a component of human bone. HAp films have been fabricated by various dry and wet processes, such as plasma spraying, ${ }^{7-10)}$ thermal

${ }^{* 1}$ Corresponding author, E-mail: hieda@mech.titech.ac.jp

${ }^{* 2}$ Graduate Student, Tohoku University spraying, ${ }^{11)}$ sputtering, ${ }^{12,13)}$ chemical vapor deposition, ${ }^{14)}$ the sol-gel method, ${ }^{15-18)}$ and others. Among these processes, the sol-gel methods achieves good adhesion of HAp films on the surface of implants made of $\mathrm{Ti}$ and $\mathrm{Ti}$ alloys, for which a long-term usage is required. The adhesive strength of HAp films on the surface of implants have been evaluated by various methods according to ASTM C633, $\left.{ }^{9}\right)$ ASTM F1147$05,{ }^{7)}$ and ISO $4624 .{ }^{11)}$ There are many researches who have studied on the adhesive strength of HAp films, but only few researches have studied on the effect of the body environment on the adhesive strength of HAp films. Water, inorganic ions, and proteins present in the human body may lead to the deterioration of HAp films. Thus, the investigation of the effect of the body environment on the adhesive strength of HAp films fabricated on the surface of the metallic implant is very important for biomedical applications. The adhesion between sol-gel fabricated HAp films and TNTZ before and after immersion in Ringer's solution was evaluated in this study. In addition, the effect of the surface morphology of TNTZ on the adhesion of the sol-gel fabricated HAp films was also investigated.

\section{Experimental Procedure}

\subsection{Preparation of TNTZ disks and evaluation of their surface morphologies}

A hot-forged bar of TNTZ (Nb: 29.2, Ta: 13.0, Zr: 4.66, C: $0.011, \mathrm{O}: 0.076, \mathrm{~N}: 0.007$, and Ti: balance; mass\%) with a diameter of $24 \mathrm{~mm}$ was used as raw material. For this raw material, the thermomechanical treatment schematically shown in Fig. 1 was carried out. Namely, the original TNTZ bar was transformed into a square bar of $15 \mathrm{~mm} \times 15 \mathrm{~mm}$ by hot forging at $1273 \mathrm{~K}$ in argon atmosphere, followed by air cooling. The hot-forged TNTZ bar was hot-rolled to a diameter of $12 \mathrm{~mm}$, again at $1273 \mathrm{~K}$ in argon atmosphere and followed by air cooling. The hot-rolled bar was machined 


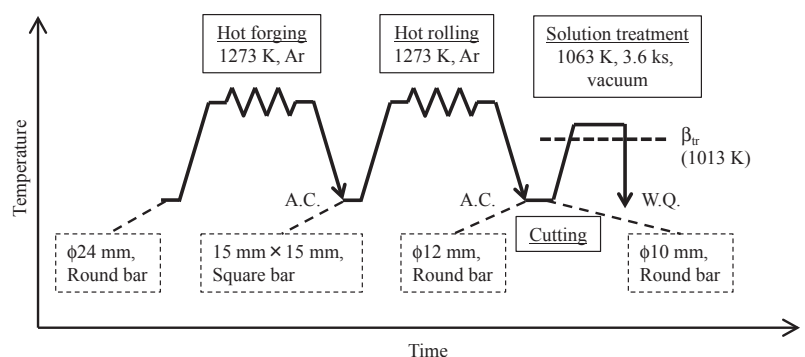

Fig. 1 Schematic drawing of thermomechanical treatment carried out on TNTZ.

into a diameter of $10 \mathrm{~mm}$ using a lathe. After machining, a solution treatment was performed on the TNTZ bar at $1063 \mathrm{~K}$, which is $50 \mathrm{~K}$ above its $\beta$-transus temperature of TNTZ, for $3.6 \mathrm{ks}$ in vacuum, followed by ice-water quenching. After the solution treatment, TNTZ disks of $10 \mathrm{~mm}$ in diameter and $2 \mathrm{~mm}$ in thickness were cut from the TNTZ bar. The TNTZ disks were wet-polished using waterproof abrasive papers of up to \#4000 grit and then buffpolished with a colloidal silica dispersion. Some of the mirror-polished TNTZ disks were polished using an waterproof abrasive paper of \#600. These TNTZ disks are denoted by mechanically polished TNTZ disks hereinafter. After polishing, the TNTZ disks were cleaned ultrasonically in acetone for $10 \mathrm{~min}$. The surfaces of mirror-polished and mechanically polished TNTZ disks were observed using a scanning electron microscope (SEM) equipped with an energy dispersive X-ray spectroscope analyzer (EDS) and atomic force microscope (AFM). In addition, the crosssectional profiles of these TNTZ disks were observed and their surface roughness was determined using an AFM.

\subsection{Fabrication of hydroxyapatite films}

HAp films were fabricated on mirror-polished and mechanically polished TNTZ disks by a sol-gel process. ${ }^{17}$ Deionized water was added to the mixture of $5.2 \mathrm{~mL}$ of triethyl phosphate and $11.47 \mathrm{~mL}$ of dehydrated ethanol at a [water]/[P] molar ratio of 12 under $\mathrm{N}_{2}$ atmosphere and, then, the precursor solution was stirred for $3 \mathrm{~d}$. Calcium nitride tetrahydrate in dehydrated ethanol was added to the precursor solution at a stoichiometric $[\mathrm{Ca}] /[\mathrm{P}]$ ratio of 1.67 and subsequently stirred for $1 \mathrm{~h}$. After that, the precursor solution was kept for $5 \mathrm{~d}$ at room temperature. The precursor solution was spin-coated onto TNTZ disks at a rotation speed of $6500 \mathrm{rpm}$, followed by drying at $353 \mathrm{~K}$ for $2 \mathrm{~h}$ and subsequent calcination at $823 \mathrm{~K}$ for $2 \mathrm{~h}$ in the atmosphere.

\subsection{Analysis of fabricated hydroxyapatite films}

The phase constituents of the calcined films were identified using an X-ray diffraction (XRD). $\mathrm{Cu} \mathrm{K} \alpha$ radiation was employed as X-ray source. The voltage and the current were $40 \mathrm{kV}$ and $150 \mathrm{~mA}$, respectively. The observation and analysis of the surface was performed using an SEM. The accelerating voltage was $15 \mathrm{kV}$. Some of the samples were immersed in Ringer's solution at $310 \mathrm{~K}$ for $7 \mathrm{~d}$ in an incubator. The surface of the HAp films fabricated on the TNTZ disks before and after immersion in Ringer's solution was observed using an SEM.

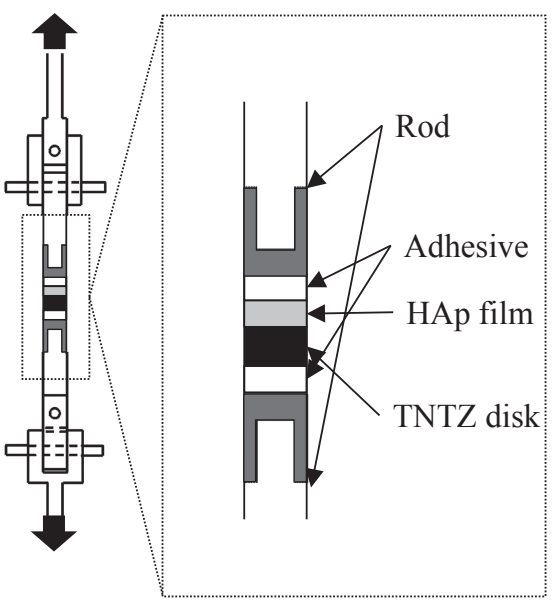

Fig. 2 Schematic drawing of apparatus used for adhesion tests.

\subsection{Adhesion tests}

The adhesive strengths of HAp films fabricated on mirrorpolished and on mechanically polished TNTZ disks were evaluated by adhesion tests according to ASTM F 1147-05. ${ }^{7}$ Schematic drawings of the tools used for the adhesion tests are schematically shown in Fig. 2. The samples were fixed between stainless steel rods $(\phi 10 \mathrm{~mm})$ at an applied load of $0.138 \mathrm{MPa}$ using a polymethylmethacrylate-based commercial dental adhesive (Super-bond, Sun Medical Co., Ltd., Japan). The samples and rods were set in the Instron-type testing machine with a maximum load capacity of $20 \mathrm{kN}$ and pulled vertically with a cross-head speed of $4.17 \times 10^{-5}$ $\mathrm{m} \cdot \mathrm{s}^{-1}$. The adhesive strength $(\sigma)$ was calculated using the following equation:

$$
\sigma=F / A
$$

Where $F$ and $A$ are the total load applied until fracture occurs and the contact area between the TNTZ disk and the rod, respectively. The tensile strength of the adhesive, which indicates the upper limit of the present adhesion tests, was also measured by fixing the mechanically polished TNTZ disk between the rods using this adhesive. The result is $29.7 \pm 3.5 \mathrm{MPa}$ (around 30 MPa). A statistical analysis of the adhesive strength was carried out by means of Student's $t$-test.

\subsection{Fracture surface analysis}

After the adhesion test, the fracture surfaces of both sides of the samples were observed by SEM and analyzed by EDS. Carbon was deposited on the fracture surfaces to prevent charging-up. The fracture area at the interface between HAp and TNTZ was evaluated by using Image J. ${ }^{19)}$ The fracture area was defined as the area where fracture occurred, normalized by the whole area of the TNTZ disks. A statistical analysis of the fracture area was carried out by means of Student's $t$-test.

\section{Results and Discussion}

\subsection{Surface morphologies of mirror-polished and me- chanically polished TNTZ disks}

Figure 3 shows SEM and AFM images of surfaces of the mirror-polished ((a), (c)) and the mechanically polished 


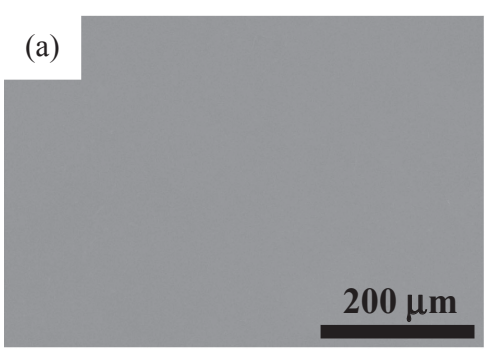

(c)

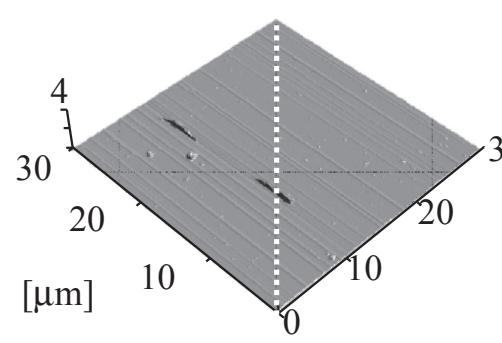

(e)

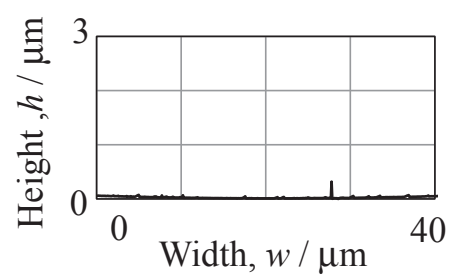

(b)

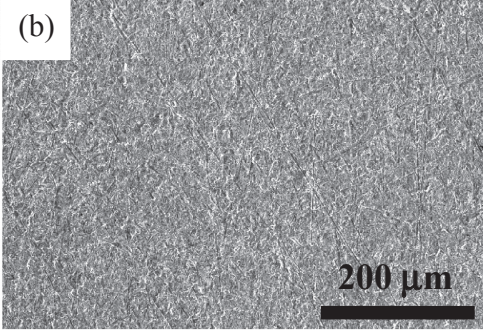

(d)

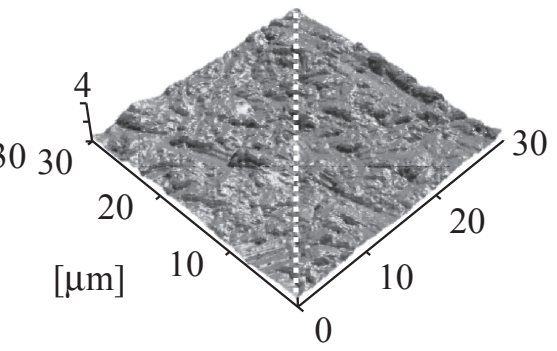

(f)

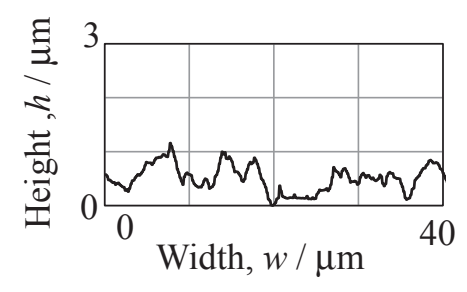

Fig. 3 SEM and AFM images of (a), (c) mirror-polished and (b), (d) mechanically polished TNTZ surfaces, respectively. Cross-sectional profiles of (e) mirror-polished and (f) mechanically polished TNTZ surfaces corresponding to white dashed lines in (c) and (d), respectively.

TNTZ disks ((b), (d)). Figure 3(e) and (f) also show crosssectional profiles, namely relationship between height profile and width corresponding to the dashed lines drawn in the AFM image of (c) and (d), respectively. The SEM image shows much larger area than that of the micrograph acquired by AFM. An apparent difference in the surface morphology is observed between the mirror-polished and the mechanically polished TNTZ disks. The mirror-polished TNTZ disk exhibits a smooth surface and its average surface roughness is approximately $16 \mathrm{~nm}$. The surface of the mechanically polished TNTZ disk has uniform asperities with heights of less than $1 \mu \mathrm{m}$. The average surface roughness of the mechanically polished TNTZ disk is approximately $187 \mathrm{~nm}$.

\subsection{Phase constituents and surface morphologies of hydroxyapatite films}

A typical XRD pattern of the HAp film fabricated on the mirror-polished TNTZ disk by the sol-gel process is shown in Fig. 4 . The peak at around $38^{\circ}$ corresponds to the (110) plane of $\beta$-Ti. The peaks attributed to HAp are present at around $26^{\circ}$ and $32-36^{\circ}$. Thus, it is confirmed that HAp is successfully formed on the TNTZ disk. Similar HAp was also obtained in the case of fabrication on the mechanically polished TNTZ disks. A peak attributable to $\mathrm{CaCO}_{3}$ is also present in the XRD pattern of the HAp film. In addition, the peaks attributed to $\mathrm{TiO}_{2}$, which was formed by oxidation of the surface of the TNTZ disk during calcination, are also observed in the XRD pattern.

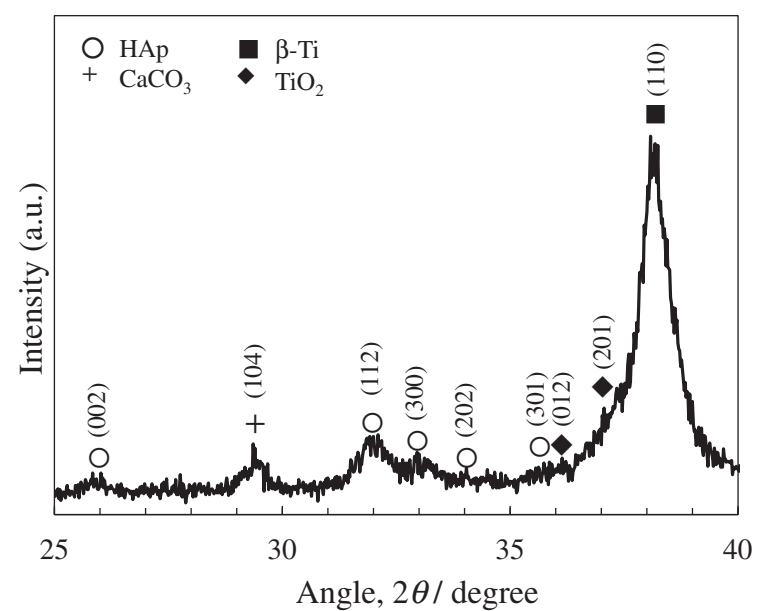

Fig. 4 Typical XRD pattern of HAp film fabricated on mirror-polished TNTZ disk by sol-gel process.

Figure 5 shows SEM images of the center and the edge parts of HAp films fabricated on the mirror-polished and the mechanically polished TNTZ disks. The average thicknesses of the HAp films fabricated on the mirror-polished TNTZ are about $179 \mathrm{~nm}$ and $325 \mathrm{~nm}$ at the center and the edge parts, respectively. The thickness of the HAp film at the edge part is greater than that at the center part because the centrifugal force carries residual solution to the edge of the TNTZ disk and surface tension prevents the solution from spinning off during the spin-coating process. At the center part of the HAp 

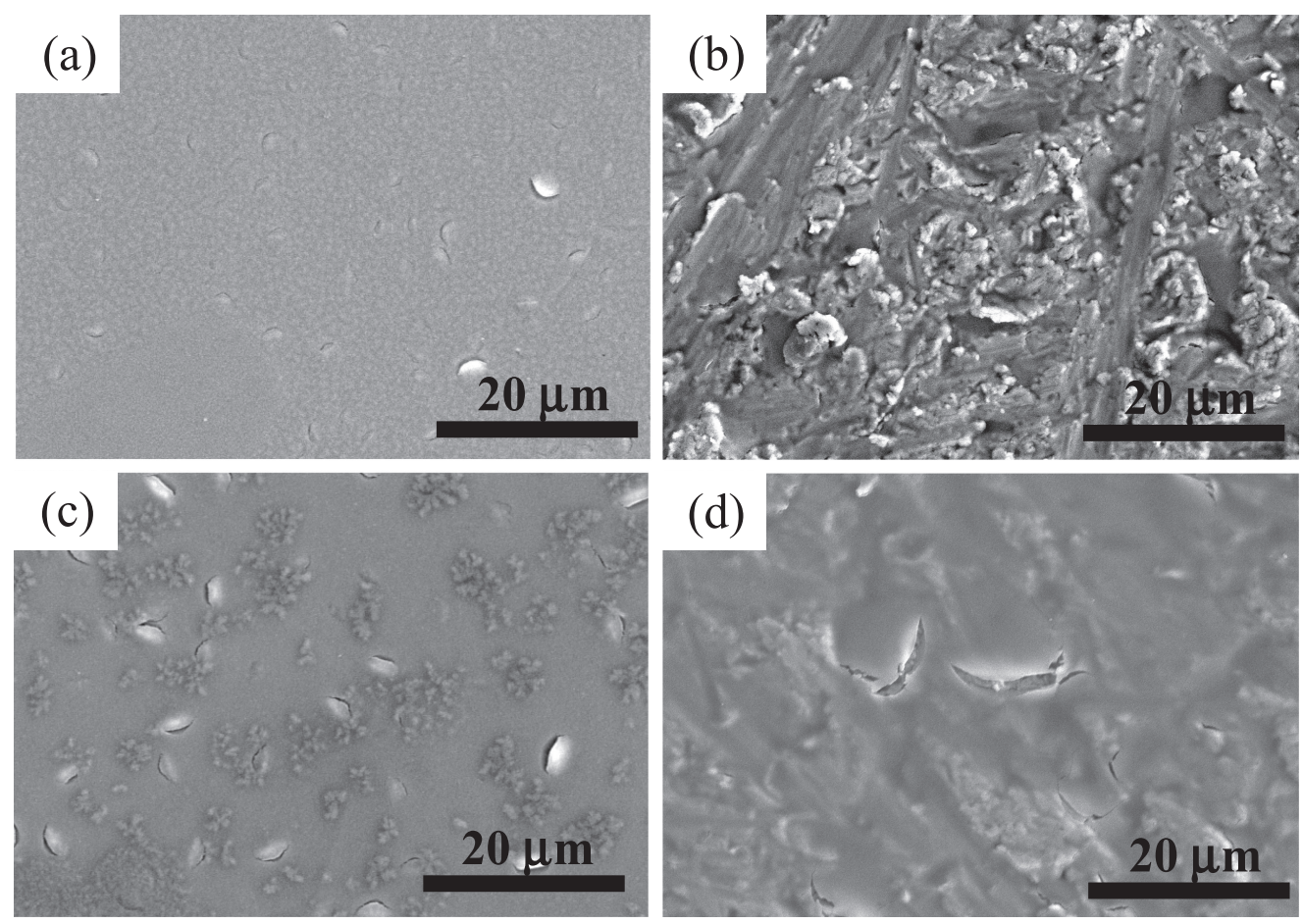

Fig. 5 SEM images of central parts of HAp films fabricated on (a) mirror-polished and (b) mechanically polished TNTZ disks, and images of edge parts for (c) mirror-polished and (d) mechanically polished TNTZ disks before immersion in Ringer's solution.

film on the mirror-polished TNTZ disk (Fig. 5(a)), the surface of HAp film has less asperity in comparison with the HAp film fabricated on the mechanically polished TNTZ disk (Fig. 5(b)). Many cracks are observed at the edge part of the surface of the HAp film deposited on the mirror-polished TNTZ disk as shown in Fig. 5(c). It is supposed that these cracks are generated during the calcination process. In the case of the HAp film being fabricated on the mechanically polished TNTZ disk, the surface asperities of the center part of the disk remain because the HAp film is thin and covers the surface of the TNTZ disk along its asperities (Fig. 5(b)). On the other hand, surface asperities are hardly observed at the edge part of the HAp film fabricated on the mechanically polished TNTZ disk (Fig. 5(d)). The average thicknesses of the HAp films fabricated on the mechanically polished TNTZ are about $575 \mathrm{~nm}$ and $649 \mathrm{~nm}$ at the center and the edge parts, respectively. Cracks are also observed at the edge part of the HAp film fabricated on the mechanically polished TNTZ disk.

\subsection{Adhesive strengths of hydroxyapatite films}

Figure 6 shows the adhesive strengths of HAp films fabricated on the mirror-polished and the mechanically polished TNTZ disks before and after immersion in Ringer's solution at $310 \mathrm{~K}$ for $7 \mathrm{~d}$. As stated above, the average tensile strength of the adhesive is around $30 \mathrm{MPa}$, which represents the limit value of the adhesive strength measurable by this test. The average adhesive strengths of the HAp films fabricated on the mirror-polished TNTZ disk before and after immersion in Ringer's solution are about $16.0 \mathrm{MPa}$ and $21.5 \mathrm{MPa}$, respectively. On the other hand, the average adhesive strengths of the HAp films fabricated on the mechanically polished TNTZ disks before and after immersion in Ringer's solution are about $23.2 \mathrm{MPa}$ and $21.2 \mathrm{MPa}$,

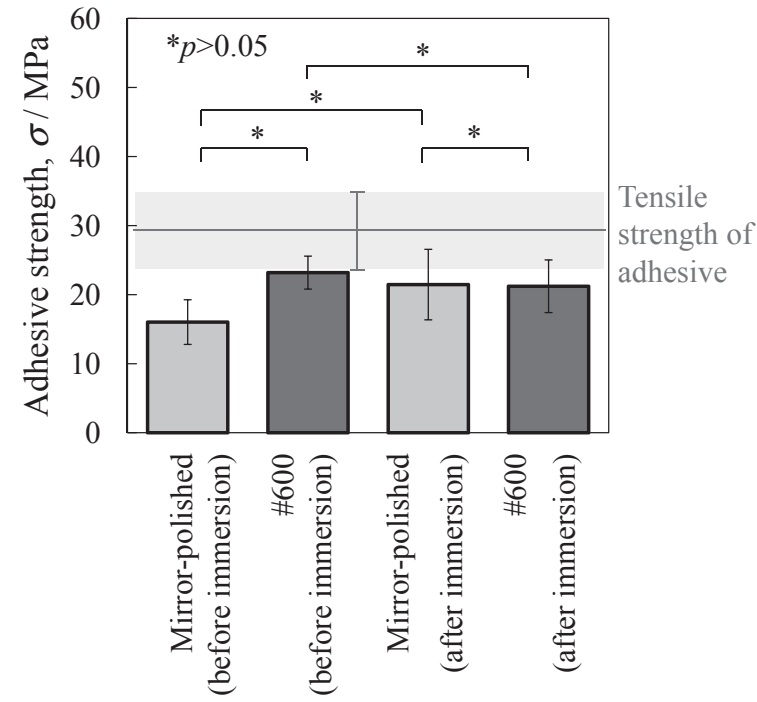

Fig. 6 Adhesive strengths of HAp films fabricated on mirror-polished and mechanically polished TNTZ disks before and after immersion in Ringer's solution at $310 \mathrm{~K}$ for $7 \mathrm{~d}$.

respectively. Therefore, these values show no significant differences $(p>0.05)$. Thus, there is no difference in the adhesive strength of HAp films with different surface morphology. Moreover, the adhesive strength exhibits similar values before and after immersion in Ringer's solution at $310 \mathrm{~K}$ for $7 \mathrm{~d}$.

\subsection{Fracture surfaces of hydroxyapatite films after adhesion tests}

As schematically shown in Fig. 7, four types of fractures may occur as a result of the adhesion tests: fracture inside the adhesive (Fig. 7(a)), fracture at the interface between adhesive and HAp (Fig. 7(b)), fracture inside HAp 
(a)

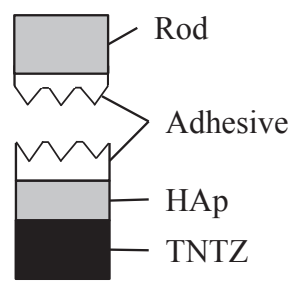

(c)

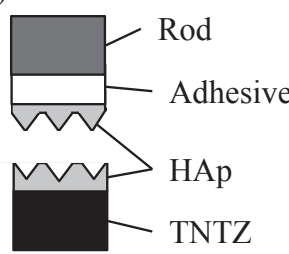

(b)

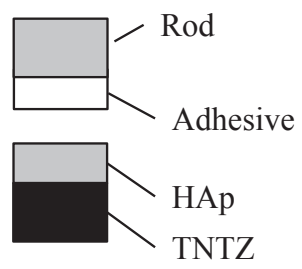

(d)

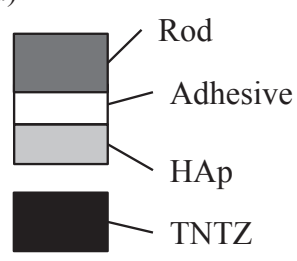

Fig. 7 Schematic explanation of different fracture types occurring during adhesion tests: (a) fracture inside adhesive, (b) fracture at interface between adhesive and HAp, (c) fracture inside HAp, and (d) fracture at interface between HAp and TNTZ.

(Fig. 7(c)), and fracture at the interface between HAp and TNTZ (Fig. 7(d)). Fracture occurs at the weakest part of a sample. If the interaction between the adhesive and HAp is weak, fracture occurs easily at the interface. Therefore, the selection of a suitable adhesive is important. Actually, the occurring fracture is a combination of the four different fracture types as already shown in Fig. 7. The fracture type is identified on the basis of EDS maps of $\mathrm{C}, \mathrm{Ca}$, and $\mathrm{Ti}$ of the fracture surfaces of the sample and opposite sides. For example, if $\mathrm{C}$ is detected at both sides of the sample, this region corresponds to fracture inside the adhesive, as already schematically shown in Fig. 7(a). The region where $\mathrm{Ca}$ is detected at the sample side and $\mathrm{C}$ is detected at the opposite side corresponds to fracture at the interface between adhesive and HAp (Fig. 7(b)). On the other hands, if $\mathrm{Ca}$ is detected at both sides of the sample, this region corresponds to fracture inside the HAp (Fig. 7(c)). Fracture occurs at the interface between HAp and TNTZ if Ti is detected at the fracture surface of the sample side and $\mathrm{Ca}$ is detected at the opposite surface, as already schematically shown in Fig. 7(d).

Figure 8 shows the fracture surfaces and corresponding EDS maps of sample ((a), (c)) and opposite sides ((b), (d)) of an HAp film on the mirror-polished TNTZ disk before ((a), (b)) and after the immersion in Ringer's solution ((c), (d)) after the adhesion tests. Regions in which $\mathrm{C}$ is detected at both sides and regions in which $\mathrm{Ca}$ is detected at the sample side and $\mathrm{C}$ is detected at the opposite side are observed for the majority of the fracture surfaces before and after immersion in Ringer's solution. This result suggests that most parts of the samples are fractured inside of the adhesive or at the interface between adhesive and HAp film, which causes no significant differences in the adhesive strength before and after immersion in Ringer's solution, as already shown in Fig. 6. Regions in which $\mathrm{Ca}$ is not detected at the sample side and $\mathrm{Ca}$ is detected at the opposite side are also present before and after immersion in Ringer's solution. Fracture at the interface between HAp film and mirrorpolished TNTZ disk also occurs.

The fracture surfaces of sample ((a), (c)) and opposite sides ((b), (d)) of a HAp film deposited on the mechanically polished TNTZ disk before ((a), (b)) and after immersion in Ringer's solution ((c), (d)) are shown in Fig. 9. Like in the case of the HAp film on the mirror-polished TNTZ disk, regions corresponding to fracture inside the adhesive and at the interface between adhesive and HAp film are observed for most parts of the samples before and after immersion in Ringer's solution. However, the observed regions in which $\mathrm{Ca}$ is detected at both sides of the fracture surface indicate that fracture occurs inside of the HAp film. No region corresponding to fracture at the interface between HAp film and the mechanically polished TNTZ disk is present. Fracture mainly occurs inside of the adhesive or at the interface between the adhesive and the HAp film. Therefore, there are no significant differences in the adhesive strengths before and after immersion in Ringer's solution. In addition, the adhesive strengths show no difference in comparison to those of the HAp films fabricated on the mirror-polished TNTZ disks before and after immersion in Ringer's solution.

In order to evaluate the adhesion of HAp films deposited on the mirror-polished and the mechanically polished TNTZ disks, the fracture areas at the interface between HAp and TNTZ were measured on the basis of SEM images before and after immersion in Ringer's solution. Figure 10 shows binary images of representative fracture surfaces of HAp films fabricated on mirror-polished TNTZ before and after immersion in Ringer's solution. The samples are fractured at the interface between HAp and TNTZ before and after immersion in Ringer's solution, reflected by the white regions in Fig. 10, whereas the black regions indicate the other fracture types as shown in Fig. 7(a), (b) and (c).

The average fracture areas at the interface between HAp films and the mirror-polished and mechanically polished TNTZ disks are shown in Fig. 11. The average fracture areas at the interface between HAp films and mirror-polished TNTZ disks before and after immersion in Ringer's solution are about $3.9 \%$ and $23 \%$, respectively, revealing a significant increase after immersion in Ringer's solution. As already shown in Fig. 9, no fracture at the interface between HAp film and the mechanically polished TNTZ disk is observed, both before and after immersion in Ringer's solution. These results indicate that the HAp film on the mechanically polished TNTZ disk exhibits greater adhesion in comparison with the HAp film on the mirror-polished TNTZ. The average adhesive strength between HAp film and the mechanically polished TNTZ is more than around $20 \mathrm{MPa}$ and is maintained at the same level even after immersion in Ringer's solution as shown in Fig. 6. On the other hand, the adhesive strength of the HAp film fabricated on the mirror-polished TNTZ is lower than that of the HAp film fabricated on the mechanically polished TNTZ because a fracture occurred at the interface between HAp and TNTZ. The higher surface roughness increases the adhesive strength of the HAp film. It is supposed that anchor effect caused by surface asperity works to increase the adhesive strength of HAp film.

As already shown in Fig. 10, the fracture at the interface between HAp and TNTZ most occurs at the edge and middle parts of the HAp films after immersion in Ringer's solution. 

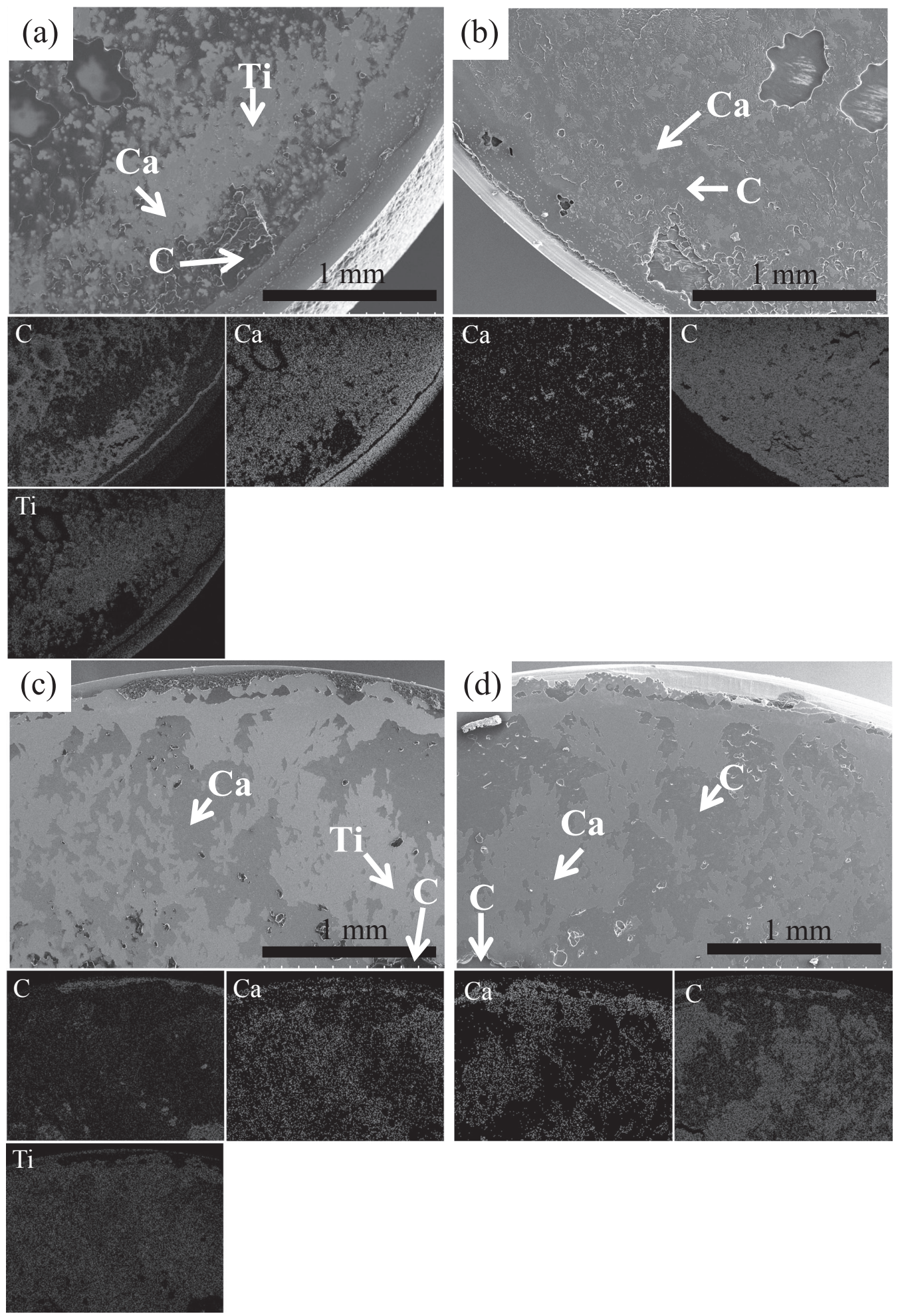

Fig. 8 SEM images and EDS maps of fracture surfaces of HAp films fabricated on mirror-polished TNTZ disks at (a) sample side and at (b) opposite side before immersion in Ringer's solution, and at (c) sample side and at (d) opposite side after immersion in Ringer's solution at $310 \mathrm{~K}$ for $7 \mathrm{~d}$.

This behavior seems to arise from the coalescence of fracture regions that exist before immersion in Ringer's solution. Many cracks are observed at the edge and middle parts of the surfaces of HAp films fabricated on mirror-polished TNTZ. It is supposed that the Ringer's solution penetrates the inside of the HAp films along cracks generated during calcination and, thereby, connects regions with weak adhesion between HAp and TNTZ. These regions with weak adhesion between HAp and TNTZ are caused by thermal stress during calcination in the case of HAp films deposited on mirror-polished TNTZ. HAp films fabricated on mechanically polished TNTZ also contains cracks at the edge part, as already shown in Fig. 5(d). It is supposed that the surface asperities prevent the formation of regions with weak adhesion between HAp and TNTZ during the calcination process. Strong adhesion is maintained between HAp and TNTZ although many cracks 

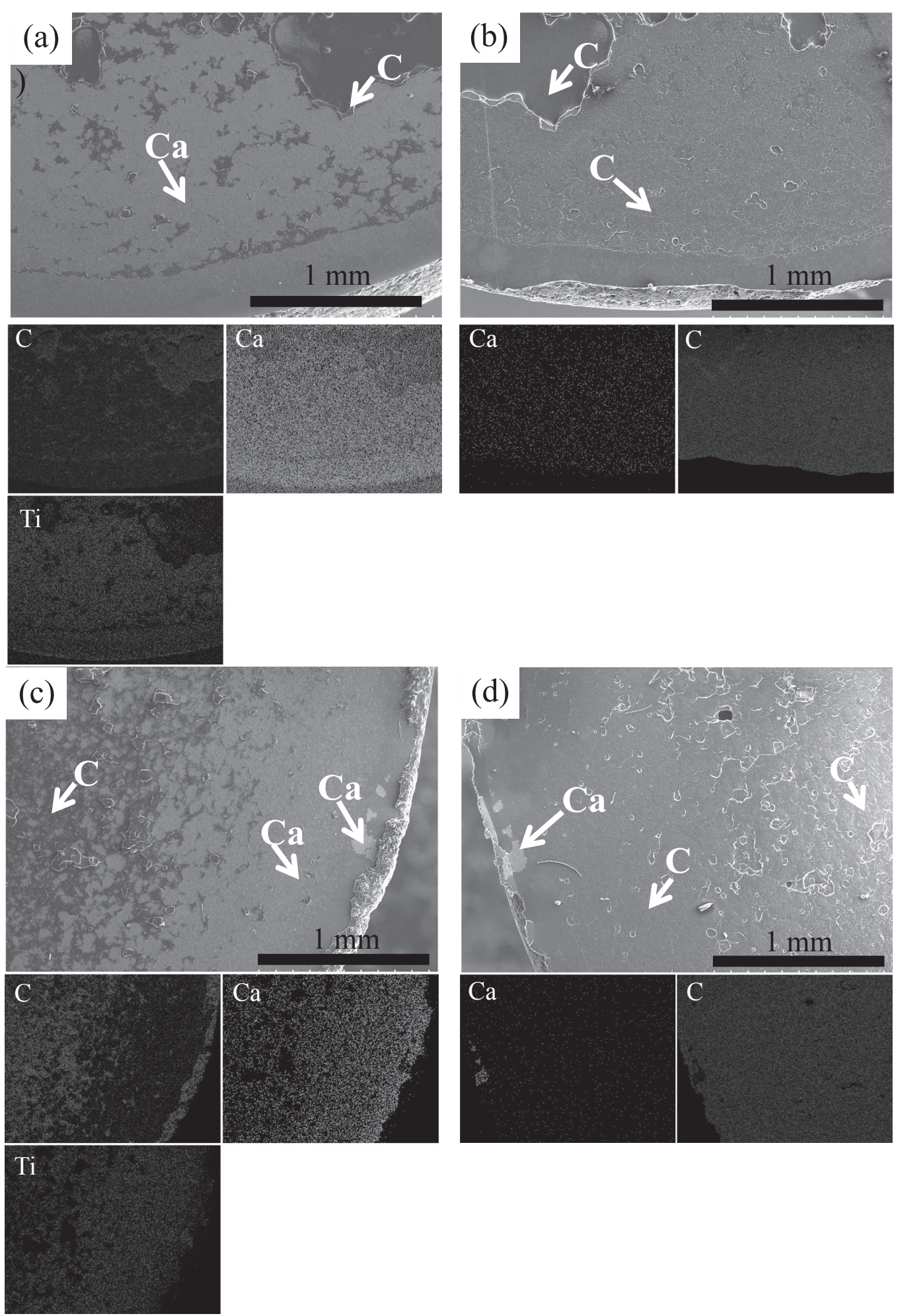

Fig. 9 SEM images and EDS maps of fracture surfaces of HAp films fabricated on mechanically polished TNTZ disks at (a) sample side and at (b) opposite side before immersion in Ringer's solution, and at (c) sample side and at (d) opposite side after immersion in Ringer's solution at $310 \mathrm{~K}$ for $7 \mathrm{~d}$.

occur in the HAp film fabricated on mechanically polished TNTZ.

\section{Conclusions}

A biomedical $\beta$-type titanium alloy, Ti-29Nb-13Ta-4.6Zr alloy (TNTZ), was coated with HAp through a sol-gel and a spin coating process in order to improve its hard-tissue compatibility. The adhesive strengths of HAp films fabricated on mirror-polished and on mechanically polished TNTZ were evaluated by adhesion tests according to ASTM F1147-05 before and after immersion in Ringer's solution at a temperature of $310 \mathrm{~K}$ for $7 \mathrm{~d}$. Images of the fracture areas of HAp films at the interface between HAp and TNTZ were taken by SEM and $\mathrm{C}, \mathrm{Ca}$, and $\mathrm{Ti}$ elemental maps were acquired by EDS. The following results were obtained. 
(a)

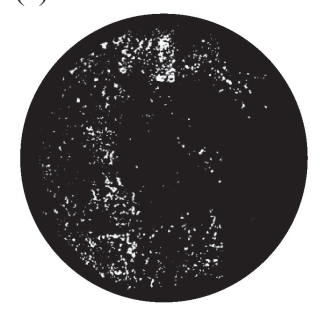

(b)

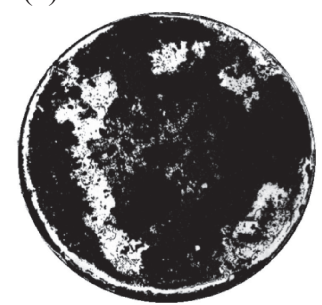

$10 \mathrm{~mm}$

Fig. 10 Typical binary images of SEM fracture surfaces showing white regions where fracture occurred at interface between HAp films and mirror-polished TNTZ (a) before and (b) after immersion in Ringer's solution.

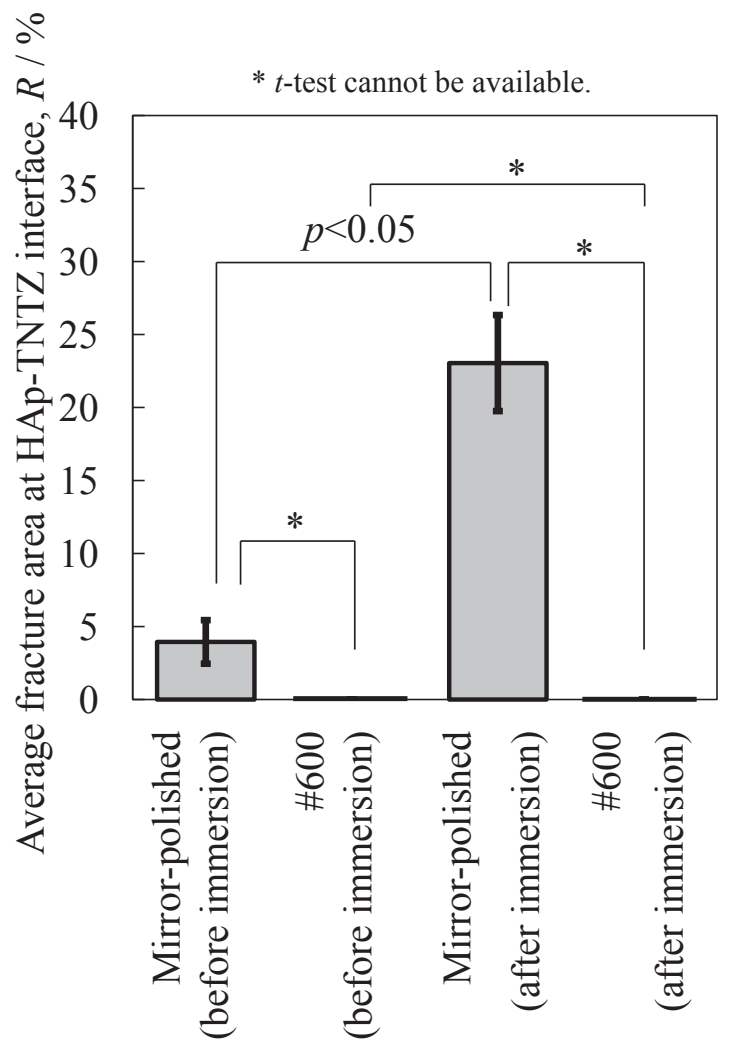

Fig. 11 Average fracture areas at the interface between HAp films and mirror-polished and mechanically polished TNTZ disks before and after immersion in Ringer's solution at $310 \mathrm{~K}$ for $7 \mathrm{~d}$.

(1) For most parts of the samples, fracture of the HAp films occurs inside of the adhesive and at the interface between adhesive and HAp, which leads to no significant differences in the adhesive strengths of the HAp films fabricated on mirror-polished and mechanically polished TNTZ before and after immersion in Ringer's solution.

(2) The fracture area at the interface between HAp and mirror-polished TNTZ increases from $3.9 \%$ to $23 \%$ after immersion in Ringer's solution. On the other hand, no fracture occurs at the interface between HAp and mechanically polished TNTZ before and after immersion in Ringer's solution. These results suggest that

HAp films fabricated on mechanically polished TNTZ exhibit greater adhesion than that on mirror-polished TNTZ. In addition, it is found that the measurement of the fracture area is important to evaluate the adhesion of HAp films.

(3) It is supposed that Ringer's solution penetrates along cracks that are formed in the HAp films during the calcination process and regions with weak adhesion between HAp and TNTZ are connected during immersion in Ringer's solution in the case of HAp films fabricated on mirror-polished TNTZ.

\section{Acknowledgments}

This study was supported in part by a Grant-in-Aid for Scientific Research from the Japan Society for the Promotion of Science (Grant Numbers 24246111 and 25820348). This study was also funded by the Tohoku Leading Women's JUMP UP Project, Tohoku University; the inter university cooperative research program "Innovative Research for Biosis-Abiosis Intelligent Interface," by the Ministry of Education, Culture, Sports, Science and Technology of Japan; and the cooperative research and development center for Advanced Materials, Institute for Materials Research, Tohoku University.

\section{REFERENCES}

1) M. Niinomi: J. Mech. Behav. Biomed. Mater. 1 (2008) 30-42.

2) H. J. Rack and J. I. Qazi: Mater. Sci. Eng. C 26 (2006) 1269-1277.

3) D. Kuroda, M. Niinomi, M. Morinaga, Y. Kato and T. Yashiro: Mater. Sci. Eng. A 243 (1998) 244-249.

4) M. Niinomi, M. Nakai and J. Hieda: Acta Biomater. 8 (2012) 38883903.

5) R. Adell, U. Lekholm, B. Rockler and P. I. Brånemark: Int. J. Oral Surg. 10 (1981) 387-416.

6) T. Albrektsson, P. I. Brånemark, H. A. Hansson and J. Lindström: Acta Orthop. Scand. 52 (1981) 155-170.

7) G. Singh, S. Singh and S. Prakash: Surf. Coat. Tech. 205 (2011) 48144820 .

8) L. Sun, C. C. Berndt, K. A. Gross and A. Kucuk: J. Biomed. Mater. Res. A 58 (2001) 570-592.

9) F. J. Xiao, L. Peng, Y. Zhang and L. J. Yun: J. Mater. Sci.: Mater. Med. 20 (2009) 1653-1658.

10) X. Zheng, M. Huang and C. Ding: Biomater. 21 (2000) 841-849.

11) R. Gadow, A. Killinger and N. Stiegler: Surf. Coat. Tech. 205 (2010) 1157-1164.

12) W. Chen, Y. Liu, H. S. Courtney, M. Bettenga, C. M. Agrawal, J. D. Bumgardner and J. L. Ong: Biomater. 27 (2006) 5512-5517.

13) K. van Dijk, H. G. Schaeken, J. C. G. Wolke, C. H. M. Marée, F. H. P. M. Habraken, J. Verhoeven and J. A. Jansen: J. Biomed. Mater. Res. A 29 (1995) 269-276.

14) J. A. Darr, Z. X. Guo, V. Raman, M. Bououdina and I. U. Rehman: Chem. Commun. 6 (2004) 696-697.

15) H. W. Kim, Y. H. Koh, L. H. Li, S. Lee and H. E. Kim: Biomater. 25 (2004) 2533-2538.

16) T. A. Kuriakose, S. N. Kalkura, M. Palanichamy, D. Arivuoli, K. Dierks, G. Bocelli and C. Betzel: J. Cryst. Growth. 263 (2004) 517523.

17) L. H. Li, H. W. Kim, S. H. Lee, Y. M. Kong and H. E. Kim: J. Biomed. Mater. Res. A 73A (2005) 48-54.

18) D. M. Liu, T. Troczynski and W. J. Tseng: Biomater. 22 (2001) 17211730 .

19) C. A. Schneider, W. S. Rasband and K. W. Eliceiri: Nature Methods 9 (2012) 671-675. 